\title{
Impact des facteurs individuels et environnementaux sur le taux d'aberrations chromosomiques de type translocations Partie 2 : agents toxiques liés à une exposition professionnelle
}

\author{
E. GRÉGOIRE ${ }^{1}$, G. GRUEL ${ }^{1}$, C. MARTIN ${ }^{1}$, S. ROCH-LEFÈVRE $^{1}$, P. VOISIN $^{1}$, \\ A. VAURIJOUX ${ }^{1}$, L. ROY ${ }^{1}$
}

(Manuscrit reçu le 13 mai 2009, accepté le 14 décembre 2009)

RÉSUMÉ Dans la première partie de cette revue, nous avons examiné, au travers d'une étude bibliographique approfondie, l'influence de différents facteurs liés à l'individu (âge, sexe, consommation d'alcool ou de tabac) sur le taux de translocations. Dans cette seconde partie, notre revue de la littérature s'est attachée à l'étude de facteurs plus toxiques liés à une exposition professionnelle. Tous les agents toxiques analysés induisent une augmentation du taux de translocations au sein des lymphocytes des personnes exposées. Cependant 2 agents (hydrocarbures aromatiques polycycliques -HAPs- et les métaux lourds) augmentent de manière significative le taux des translocations des personnes exposées. Ce type d'agents toxiques augmente le taux de translocations proportionnellement à la dose et à la durée d'exposition. La sensibilité de la technique FISH a permis de mettre en évidence la nécessité et le bon fonctionnement des équipements de protection. Pour tous les facteurs toxiques liés à une exposition professionnelle, un équipement de protection adapté et ergonomique ou bien la période d'exposition ont réduit significativement le taux de translocations des individus exposés. Ainsi la technique FISH pourrait servir d'indicateur d'exposition. En conclusion, afin d'évaluer rétrospectivement l'exposition d'un individu, il est important de connaitre le plus précisément possible son curriculum clastogène et son âge. Ainsi, un taux de translocations limite pourrait être établi en fonction de l'impact des expositions professionnelles et environnementales connues aujourd'hui et de la connaissance du passé de l'individu.

ABSTRACT Impact of genotoxic agents on the rate of translocations - part 2: toxic agents related to occupational exposure.

In the first part of this review, we examined through an extensive literature review, the influence of various factors related to the individual (age, sex, alcohol and tobacco) on the rate of translocation. In the second part, our literature review focused on the study of more toxic factors related to occupational exposure. All toxic agents tested induced an increase in the translocation rate in lymphocytes of exposed people. However 2 agents (polycyclic aromatic hydrocarbons -PAHs- and heavy metals) increase significantly the rate of translocation of exposed persons. This increase is function to the dose and duration of exposure. The sensitivity of FISH technique has highlighted the necessity of protective equipment. For all the toxic

\footnotetext{
1 IRSN, Laboratoire de Dosimétrie Biologique, BP 17, 92262 Fontenay-aux-Roses Cedex, France.
} 


\section{E. GRÉGOIRE et al.}

factors related to occupational exposure, protective equipment or exposure conditions reduced significantly the translocations rate of exposed subjects. Thus the FISH technique could be an exposure warning indicator. In conclusion, it is important to know as well the personnel and occupational curriculum of an individual if a retrospective dose assessment is required. Thus, the rate of translocations threshold will be based on the lifestyle rate.

Keywords: Chromosomal translocation / lifestyle factor / genotoxic agent / retrospective dosimetry / environmental factor

\section{Introduction}

Les translocations sont des aberrations chromosomiques (AC) pouvant être induites par les rayonnements ionisants, mais aussi par d'autres facteurs. Dans la première partie de cette revue, nous avons analysé l'influence de différents facteurs liés à l'individu (âge, genre, alcool et tabac), et montré que ces paramètres doivent être pris en compte pour une analyse rétrospective des doses d'irradiation reçues par les individus. Cependant au cours de l'activité professionnelle, les individus peuvent être exposés à d'autres agents, chimiques notamment, capables d'induire également des dommages à l'ADN et donc de produire des translocations. Les principaux facteurs retenus dans cette revue ont été les pesticides, le benzène et les métaux lourds.

\section{Matériels et méthodes}

La méthodologie appliquée pour l'analyse de l'impact des agents liés à une exposition professionnelle est décrite dans la partie 1. Nous avons également utilisé les bases de données PubMed et Scopus, avec les mots clés suivants selon le type de facteurs étudiés :

("chromosomal aberration" OR "chromosomal abnormality" OR "abnormal chromosome" OR "chromosome aberration") AND Translocation AND (blood OR lymphocyte OR "peripheral blood lymphocyte" OR "peripheral blood cell") AND (clastogen* OR mutagen* OR carcinogen*) AND

Benzène et HAP :

(Benzene, OR benzol, OR butadiene, OR isoprene, OR "industry shoes", OR gasoline, OR gasolene, OR pumpman, OR "gasoline, OR station attendant", OR methylene, OR sulphur dioxide, OR air pollution, OR indoor, OR "indoor air pollution", OR "aliphatic hydrocarbons", OR glycol, OR "polycyclic aromatic hydrocarbons", OR "polynuclear aromatic hydrocarbon", OR "PAH", OR "synthetic resine". 
Métaux lourds :

AND (heavy metals OR arsenic OR beryllium OR cadmium OR chromium OR titanium OR steel OR iron OR aluminium OR manganiferous OR manganese OR mercury OR nickel OR lead OR metal salts OR silver OR gold OR zinc OR copper OR bismuth OR tin).

Dans le cas des pesticides, nous avons utilisé la revue de Bolognesi (2003) comme référence et support.

\section{Résultats}

\subsection{Effet de l'exposition aux pesticides}

Les pesticides constituent une catégorie de produits chimiques hétérogènes utilisés pour le contrôle des organismes nuisibles, des mauvaises herbes ou des maladies de plantes. Ils sont considérés comme des mutagènes chimiques potentiels. Des données expérimentales ont révélé que différents ingrédients agrochimiques pouvaient induire des mutations génétiques, des aberrations chromosomiques ou des dommages à l'ADN (Bolognesi, 2003). Ce type d'agents chimiques tient sa place dans cette étude bibliographique compte tenu du nombre de personnes potentiellement exposées par l'utilisation des pesticides, soit directement dans le cas des agriculteurs ou des jardiniers, soit indirectement dans le cas de tout individu résidant à proximité des zones d'épandage. En revanche, l'effet des pesticides sur la fréquence de translocations semble n'avoir été analysé qu'au sein de deux études.

La première étude s'est intéressée à un type particulier de pesticides; les fongicides à Ethylène Bis-DithioCarbamate (EBDC) (Steenland et al., 1997) (Tab. I). Les auteurs montrent une augmentation légèrement significative des translocations après exposition à ce type de fongicides. L'effet des EBDC sur le taux de translocations est mis en évidence aussi bien chez des applicateurs à pulvérisateur dorsal, qu'au sein de propriétaires exposés $(p<0,001)$. Les propriétaires sont présents toute l'année sur la zone d'épandage mais sont exposés à des concentrations plus faibles, tandis que les applicateurs sont les plus exposés mais seulement pendant une saison (de juin à novembre). Cependant l'âge agit comme un facteur confondant. Après un ajustement par l'âge l'augmentation des translocations chez les propriétaires devient non significative, alors que cette augmentation reste significative chez les applicateurs. Par conséquent, l'effet dose et exposition est important dans le cas des fongicides sur le taux de translocations. L'effet tabac est insignifiant vu que les individus sont de très petits fumeurs (4-5 cigarettes/jour). En revanche, les applicateurs ont été en contact avec les 
TABLEAU I

Synthèse des publications qui étudient l'impact de différents pesticides sur le taux de translocations. Taux donnés pour 1000 cellules observées.

Summary of publications investigating the impact of pesticides on translocation rate. Rate given for 1000 observed cells.

\begin{tabular}{|c|c|c|c|c|}
\hline $\begin{array}{l}\text { Référence de } \\
\text { la publication }\end{array}$ & $\begin{array}{c}\text { Taux de } \\
\text { translocations pour } \\
\text { la population } \\
\text { contrôle }\end{array}$ & $\begin{array}{c}\text { Taux de } \\
\text { translocations pour } \\
\text { la population } \\
\text { exposée }\end{array}$ & $\begin{array}{c}\text { Facteur } \\
\text { d'augmentation }\end{array}$ & $\begin{array}{l}\text { Nombre d'individus } \\
\text { dans l'étude }\end{array}$ \\
\hline $\begin{array}{l}\text { Steenland } \\
\text { et al., } 1997 \text { a c } \\
\text { (ajusté/âge) }\end{array}$ & $4,59 \pm 0,82$ & $\begin{array}{c}6,07 \pm 0,97 \\
(31 \text { applicateurs }) \\
5,44 \pm 1,00 \\
\text { (13 propriétaires) }\end{array}$ & $\begin{array}{c}1,3(p=0,05) \\
1,2(\mathrm{NS}: p=0,31)\end{array}$ & $\begin{array}{c}\text { Exposés : } \\
31 \text { applicateurs de } \\
\text { fongicides à EBDC } \\
13 \text { propriétaires } \\
\text { Contrôles : } \\
30 \text { personnes }\end{array}$ \\
\hline $\begin{array}{l}\text { Tucker et al., } \\
2003 \text { a b }\end{array}$ & $3,70 \pm 0,54(13 \mathrm{NF})$ & $2,75 \pm 0,28(10 \mathrm{NF})$ & (NS) & $\begin{array}{c}\text { Exposés : } \\
20 \text { épandeurs de Phosphine } \\
\text { Contrôles : } 26\end{array}$ \\
\hline
\end{tabular}

NS : différence non significative, F : fumeurs, NF : non-fumeurs. Steenland : Translocations observé par FISH. Données en Mean \pm standard error. Tucker : Translocation observé par FISH. Donné en Mean \pm SE. ${ }^{a}$ Les taux sont ramenés à l'ensemble du génome par la formule de Lucas. ${ }^{b}$ Moyenne des fréquences génomiques $( \pm$ erreur standard) données pour 100 cellules observées au sein de la publication. Nombre de translocations non donné. ${ }^{\mathrm{c}}$ Valeurs données pour 500 cellules équivalentes ( 1500 cellules observé). Moyenne $\pm \mathrm{SE}$ (standard error). Nombre de translocations non donné. EBDC : Ethylène Bis-DithioCarbamate. * Ratio du taux population exposée sur le taux population contrôle.

produits chimiques de façon importante, au niveau de la peau, lors de la préparation du mélange de fongicides à épandre. Aucune protection des travailleurs n'est indiquée, ce qui pourrait expliquer le taux de translocations élevé.

La seconde étude montre que la dispersion d'un seul type de pesticide (la phosphine) n'induit pas d'augmentation du taux de translocations chez les épandeurs. en ayant bien pris en compte les facteurs confondants tels que l'âge et le tabac (Tucker et al., 2003). En effet un léger effet synergique du tabac et de la phosphine est montré entre les épandeurs non-fumeurs et fumeurs mais la différence n'est pas significative. Dans cette étude, les auteurs ont observé peu de translocation pour tous les sujets au contraire d'études préliminaires sur la même population dans laquelle les applicateurs de phosphine présentaient des niveaux significativement élevés d'AC stables $(p=0,003)$ (Garry et al., 1992). Lors de cette étude, les résultats du FISH ont fournit un support pour l'utilisation continuelle des équipements de protection par ces travailleurs. Ainsi, la diminution du taux de translocations dans une population exposée à ce type d'agents mutagènes a prouvée l'efficacité des protections individuelles (gants, masques, combinaisons...) (Tucker et al., 2003). Cette efficacité peut expliquer également la différence des taux de translocations entre la population exposée aux fongicides et celle exposée à la phosphine. 


\subsection{Exposition aux produits du Benzène}

Le benzène est un cancérigène connu puisqu'il induit des leucémies (Forni, 1996). L'exposition au benzène est caractérisée par de nombreuses délétions (perte totale ou partielle de matériel chromosomique) ainsi que des translocations spécifiques notamment sur les chromosomes 8, 14, 18 et, 21. Ces délétions et translocations sont celles observées par la suite au sein des cellules leucémiques en tant que marqueur de ces pathologies. C'est la raison pour laquelle la majorité des publications étudiant l'effet du benzène se sont focalisées uniquement sur l'analyse de ces chromosomes (Tab. II).

L'impact du benzène sur les translocations peut être analysé de diverses façons. Si l'étude porte sur certaines translocations spécifiques comme la $\mathrm{t}(8 ; 21)$, aberration la plus commune au sein des leucémies myéloïdes aigües (LMA) ou la $\mathrm{t}(14 ; 18)$, également présente dans des lymphomes, la tendance globale est l'augmentation significative du taux de translocation chez les personnes exposées au benzène. Par exemple, la translocation $\mathrm{t}(8 ; 21)$ est augmentée significativement que la dose soit forte $(>31 \mathrm{ppm})$ (Smith et al., 1998) ou bien très faible $(<1 \mathrm{ppm})$ (Kim et al., 2004b). Si toutes les translocations impliquant les chromosomes 8 ou 21 sont considérées, le résultat est similaire aussi bien pour les travailleurs exposés à des doses supérieures à $31 \mathrm{ppm}$ de benzène (Smith et al., 1998), que pour ceux exposés à des doses 30 fois inferieure (Kim et al., 2004a). Cependant, les travailleurs de cette dernière étude sont exposés simultanément à de nombreux autres complexes chimiques clastogènes contenus dans les émissions des fours à charbon mais le benzène reste l'agent clastogène majeur. Les groupes contrôle et exposé sont de même âge moyen (40 ans) et de même consommation tabagique ( $<20$ cigarettes par mois). Par conséquent, les effets âge et tabac ne jouent pas en facteurs confondants sur le taux de translocations. Cependant, aucune information sur la consommation d'alcool des sujets contrôles et exposés n'est mentionnée dans cette étude (Kim et al., 2004a). En revanche, l'étude de Smith et al. (1998) ne donne aucune précision sur l'âge et la consommation tabagique des individus. L'augmentation significative des AC stables a été également observée pour la translocation $\mathrm{t}(14 ; 18)$ uniquement chez des personnes fortement exposées au benzène $(>31 \mathrm{ppm})$, par rapport à celui des individus témoins $(p<0,001)$. Cependant, le même type de translocation est observé chez les gros fumeurs ( $>20$ cigarettes par jour) où des hydrocarbures aromatiques polycycliques comme le benzène, sont retrouvés (Zhang et al., 2007).

En revanche, si l'étude porte sur des chromosomes autres que ceux cités précédemment, les résultats ne montrent aucune différence significative entre le groupe exposé (< et $>31 \mathrm{ppm}$ ) et le groupe contrôle (Zhang et al., 2007). Par conséquent, le type de chromosomes peints pour la dosimétrie biologique 


\section{E. GRÉGOIRE et al.}

TABLEAU II

Synthèse des publications qui étudient l'effet de l'exposition au benzène sur le taux de translocations. Taux donnés pour 1000 cellules observées.

Summary of publications investigating the effect of benzene exposure on translocation rate. Rate given for 1000 observed cells.

Cas de translocations spécifiques

\begin{tabular}{|c|c|c|c|c|}
\hline $\begin{array}{l}\text { Référence } \\
\text { publication }\end{array}$ & $\begin{array}{c}\text { Taux de } \\
\text { translocations de la } \\
\text { population contrôle }\end{array}$ & $\begin{array}{l}\text { Taux de translocations } \\
\text { de la population } \\
\text { exposée }\end{array}$ & $\begin{array}{c}\text { Facteur * } \\
\text { d'augmentation } \\
\text { (significativité) }\end{array}$ & $\begin{array}{l}\text { Nombre total } \\
\text { d'individus }\end{array}$ \\
\hline $\begin{array}{l}\text { Kim et al., } 2004^{\mathrm{cd}} \\
\mathrm{t}(8 ; 21)\end{array}$ & - & - & $(p<0,05)$ & $\begin{array}{l}76 \text { contrôles } \\
82 \text { exposés } \\
(0,557 \text { ppm })\end{array}$ \\
\hline $\begin{array}{l}\text { Smith et al., } 1998^{\mathrm{c}} \\
\mathrm{t}(8 ; 21)\end{array}$ & - & - & $15 \quad(p<0,0001)$ & $\begin{array}{l}44 \text { contrôles } \\
\text { Exposés : } 22 \\
(>31 \mathrm{ppm})\end{array}$ \\
\hline $\begin{array}{l}\text { Zhang et al., } 2007^{\mathrm{b}} \\
\mathrm{t}(14 ; 18)\end{array}$ & $0 \pm 0,0$ & $\begin{array}{c}0 \pm 0,0(<31 \mathrm{ppm}) \\
0,49 \pm 0,25(>31 \mathrm{ppm})\end{array}$ & $\begin{array}{lr}- & \mathrm{NS}^{\mathrm{e}} \\
- & (p<0,001)^{\mathrm{e}}\end{array}$ & $\begin{array}{c}44 \text { contrôles } \\
43 \text { Exposés : } 21 \\
(<31 \mathrm{ppm}) 22 \\
\text { (> } 31 \mathrm{ppm}\end{array}$ \\
\hline
\end{tabular}

$\mathrm{t}(8 ; 21)$ indique que seules les translocations entre les chromosomes 8 et 21 ont été étudiées, - : aucune valeur n'est donnée dans la publication. ${ }^{a}$ Les taux sont ramenés à l'ensemble du génome par la formule de Lucas. ${ }^{\mathrm{b}}$ Moyenne des fréquences génomiques ( \pm erreur standard) données pour 100 cellules observées au sein de la publication. ${ }^{\mathrm{c}}$ Facteur d'augmentation donné dans la publication. ${ }^{\mathrm{d}}$ test U Mann-Whitney. ${ }^{\mathrm{e}}$ test Fisher. * Ratio du taux population exposée sur le taux population contrôle. NS : non significatif.

Cas de translocations concernant d'autres chromosomes

\begin{tabular}{|c|c|c|c|c|}
\hline $\begin{array}{l}\text { Référence } \\
\text { publication }\end{array}$ & $\begin{array}{c}\text { Taux de } \\
\text { translocations de la } \\
\text { population contrôle }\end{array}$ & $\begin{array}{c}\text { Taux de translocations } \\
\text { de la population } \\
\text { exposée }\end{array}$ & $\begin{array}{c}\text { Facteur * } \\
\text { d'augmentation } \\
\text { (significativité) }\end{array}$ & $\begin{array}{c}\text { Nombre total } \\
\text { d'individus }\end{array}$ \\
\hline $\begin{array}{l}\text { Kim et al., } 2004 \text { bc } \\
\mathrm{t}(8 ; ?)+\mathrm{t}(21 ; ?)\end{array}$ & - & - & 1,4 & $\begin{array}{l}76 \text { contrôles } \\
82 \text { exposés } \\
(0,557 \text { ppm })\end{array}$ \\
\hline $\begin{array}{l}\text { Smith et al., } \\
1998 \text { b c } \\
\mathrm{t}(8 ; ?)\end{array}$ & - & - & $\begin{array}{c}\sim 4 \\
(p<0,0001)\end{array}$ & $\begin{array}{l}44 \text { contrôles } \\
\text { Exposés }\end{array}$ \\
\hline $\begin{array}{l}\text { Smith et al., } 1998 \\
\mathrm{t}(21 ; ?)\end{array}$ & - & - & $\begin{array}{c}\sim 4 \\
(p=0,03)\end{array}$ & $22(>31 \mathrm{ppm})$ \\
\hline $\begin{array}{l}\text { Zhang et al., } \\
2007 \text { b } \\
\mathrm{t}(11 ; 4 ; 6 ; ?)\end{array}$ & $0,43 \pm 0,18$ & $\begin{array}{c}0,05 \pm 0,05(<31 \mathrm{ppm}) \\
0,61 \pm 0,38(>31 \mathrm{ppm})\end{array}$ & $\begin{array}{ll}0,12 & (\mathrm{NS})^{\mathrm{e}} \\
1,42 & (\mathrm{NS})^{\mathrm{e}}\end{array}$ & $\begin{array}{c}44 \text { contrôles } \\
43 \text { Exposés : } \\
21 \text { (<31 ppm) } \\
22(>31 \mathrm{ppm})\end{array}$ \\
\hline
\end{tabular}

?: autre chromosome que les chromosomes $8,4,6,11$ ou 2 . - : aucune valeur n'est donnée dans la publication, NS : non significatif, 4 : dans la publication, il est précisé autour de 4 (around 4-fold), ${ }^{\mathrm{e}}$ test Fisher, $*$ Ratio du taux population exposée sur le taux population contrôle.

rétrospective par la technique FISH de chromosomes est très important. Il faut éviter d'étudier les chromosomes impliqués dans les leucémies ou lymphomes dues à l'exposition au benzène. 


\section{TABLEAU III}

Synthèse des publications étudiant l'effet d'hydrocarbures aromatiques sur le taux de translocations. Taux donnés pour 1000 cellules observées.

Summary of publications investigating the effect of aromatic hydrocarbur on translocation rate. Rate given for 1000 observed cells.

\section{Exposition HAP}

\begin{tabular}{|c|c|c|c|c|c|}
\hline $\begin{array}{l}\text { Référence de la } \\
\text { publication }\end{array}$ & $\begin{array}{c}\text { Taux de } \\
\text { translocations } \\
\text { de la population } \\
\text { contrôle }\end{array}$ & $\begin{array}{c}\text { Taux de } \\
\text { translocations de la } \\
\text { population exposée }\end{array}$ & $\begin{array}{r}\text { d'a } \\
(\mathrm{Si}\end{array}$ & $\begin{array}{l}\text { acteur* } \\
\text { gmentation } \\
\text { nificativité) }\end{array}$ & Groupes analysés \\
\hline $\begin{array}{l}\text { Sram et al., } \\
2004^{\text {a b }} \text { Prague }\end{array}$ & $12,5 \pm 11,1$ & $17,2 \pm 15,7$ & 1,4 & $(p<0,05)$ & $\begin{array}{c}\text { Contrôles : } 48 \\
\text { Exposés: } 50 \text { policiers }\end{array}$ \\
\hline $\begin{array}{l}\text { Beskid et al., } \\
2007^{\text {a b }} \text { Prague" }\end{array}$ & $12,4 \pm 11,1$ & $17,2 \pm 15,7$ & 1,4 & $(\mathrm{NS})^{*}$ & $\begin{array}{l}\text { Exposés: } 50 \text { policiers } \\
\text { Contrôles : } 49\end{array}$ \\
\hline $\begin{array}{l}\text { Beskid et al., } \\
2007^{\text {a b }} \text { Kosice }\end{array}$ & $11,2 \pm 13,0$ & $15,2 \pm 11,8$ & 1,4 & $(p<0,05)^{*}$ & $\begin{array}{l}\text { Exposés: } 46 \text { policiers } \\
\text { Contrôles : } 45\end{array}$ \\
\hline $\begin{array}{l}\text { Beskid et al., } \\
2007^{\text {a b }} \text { Sofia }\end{array}$ & $8,2 \pm 7,9$ & $\begin{array}{c}16,0 \pm 9,9 \text { (policiers) } \\
12,2 \pm 8,5 \text { (bus) }\end{array}$ & $\begin{array}{l}1,9 \\
1,5\end{array}$ & $\begin{array}{r}(p<0,01)^{*} \\
(\mathrm{NS})^{*}\end{array}$ & $\begin{array}{c}\text { Exposés: } \\
26 \text { policiers }+25 \\
\text { conducteurs de bus } \\
\text { Contrôles : } 25\end{array}$ \\
\hline $\begin{array}{l}\text { Beskid et al., } \\
2007^{\text {a b }} \text { Total }\end{array}$ & $11,1 \pm 11,3$ & $15,5 \pm 12,5$ & 1,4 & $(p<0,001)^{*}$ & $\begin{array}{l}\text { Exposés : } 122 \text { policiers } \\
\text { et } 25 \text { conducteurs de bus } \\
\text { Contrôles : } 119\end{array}$ \\
\hline $\begin{array}{l}\text { Sram et al., } \\
2007^{\text {a b }} \mathrm{t}(1 ; 4)\end{array}$ & $8,5 \pm 9,5$ (mars) & $13,2 \pm 10,7$ (janvier) & 1,5 & $(p<0,01)^{*}$ & $\begin{array}{l}\text { Exposés en janvier: } \\
60 \text { policiers } \\
\text { Exposés en mars: } \\
61 \text { policiers }\end{array}$ \\
\hline
\end{tabular}

* Test U-Mann-Whitney, NS : non significatif, * taux calculé à partir des données de la publication. ${ }^{a}$ Les taux sont ramenés à l'ensemble du génome par la formule de Lucas. ${ }^{b}$ Moyenne des fréquences génomiques ( \pm erreur standard) données pour 100 cellules observées au sein de la publication. ${ }^{\mathrm{c}}$ Facteur d'augmentation donné dans la publication.

Exposition éthylbenzène + benzène

\begin{tabular}{|c|c|c|c|c|}
\hline $\begin{array}{c}\text { Référence de la } \\
\text { publication }\end{array}$ & $\begin{array}{c}\text { Taux de } \\
\text { translocations } \\
\text { de la population } \\
\text { contrôle }\end{array}$ & $\begin{array}{c}\text { Taux de } \\
\text { translocations de la } \\
\text { population exposée }\end{array}$ & $\begin{array}{c}\text { Facteur } \\
\text { d'augmentation } \\
\text { (Significativité) }\end{array}$ & Groupes analysés \\
\hline $\begin{array}{l}\text { Beskid et al., } \\
2006 \text { a b }\end{array}$ & $12,4 \pm 11,0$ & $30,6 \pm 21,1$ & (NS) & $\begin{array}{l}\text { Exposés : } 39 \\
\text { Contrôles : } 49\end{array}$ \\
\hline $\begin{array}{l}\text { Sram et al., } \\
2004^{\text {a b }}\end{array}$ & $16,3 \pm 13,0$ & $30,6 \pm 21,3$ & $(p<0,001)^{*}$ & $\begin{array}{l}\text { Exposés : } 39 \\
\text { Contrôle : } 55\end{array}$ \\
\hline
\end{tabular}

* Test U-Mann-Whitney, NS : non significatif, ${ }^{a}$ Les taux sont ramenés à l'ensemble du génome par la formule de Lucas. ${ }^{b}$ Moyenne des fréquences génomique ( \pm erreur standard) données pour 100 cellules observées au sein de la publication.

\subsection{Effet des hydrocarbures aromatiques polycycliques (émissions automobiles et industrielles)}

L'impact de la pollution de l'air contenant de fortes concentrations d'hydrocarbures aromatiques polycycliques (HAPs) sur le taux de translocations a été analysé dans 3 villes européennes (Prague, Kosice et Sofia) (Beskid et al., 2007) (Tab. III). Chaque ville possède son groupe témoin composé de personnes 
restant plus de $90 \%$ de la journée à leur domicile. Les taux observés sont significativement plus élevés chez les personnes exposées que chez des sujets nonexposés au sein des 2 villes où les concentrations aériennes en HAPs sont les plus fortes (Kosice et Sofia). À Sofia, la technique FISH a même permis de différencier les différents niveaux d'exposition. En effet, les conducteurs de bus ont un taux de translocations moins élevé que les policiers, car ils sont légèrement protégés des émanations automobiles. De même le taux de translocations varie suivant le niveau de pollution. Ainsi, la technique FISH met en évidence une différence significative des translocations entre l'hiver et le printemps chez les policiers (Sram et al., 2007). Par conséquent, l'étude des variations du taux de translocations permet une détection approfondie des doses d'exposition. L'impact de l'éthylbenzène, présent dans les gaz d'échappement des voitures, sur le taux de translocations a été également étudié (Beskid et al., 2006 ; Sram et al., 2004). La première étude ne met pas en évidence d'augmentation significative du taux de translocations chez les travailleurs exposés (Beskid et al., 2006). Tandis que l'autre étude montre une augmentation significative des translocations entre la population exposée et la population contrôle $(p<0,001)$ (Sram et al., 2004). Il semble qu'il s'agit des mêmes groupes d'études mais le test statistique n'est pas précisé chez Beskid et al. (2006). Par conséquent l'éthylbenzène semble avoir un effet sur le taux de translocations et pourrait être l'agent initiateur de ce type d'aberrations au sein des HAPs.

\subsection{Effets des métaux lourds}

Le nombre d'études portant sur les effets des métaux lourds et métalloïdes sur le taux de translocations est faible. Toutefois, nous avons tenu à présenter les effets de l'arsenic et du chrome sur le taux de translocations analysés par la technique FISH.

\section{Chrome}

Deux publications récentes montrent une augmentation significative sur le taux d'aberrations chromosomiques stables après une exposition au chrome (Tab. IV).

L'exposition au chrome concerne les personnes travaillant dans l'industrie de l'inox, notamment dans la soudure et le plaquage. L'augmentation du taux de translocations est montrée de manière significative chez des travailleurs exposés au chrome par rapport au groupe contrôle $(p<0,01)$. De plus, un effet synergique de la consommation de tabac avec l'exposition au chrome montré un taux de translocations plus élevé chez les fumeurs exposés par rapport à celui des nonfumeurs exposés $(p<0,05)$ (Maeng et al., 2004). En revanche, la consommation tabagique n'est pas indiquée. L'exposition au chrome a été aussi analysée en 
TABLEAU IV

Synthèse des publications étudiant l'effet du chrome sur le taux de translocations. Taux donné pour 1000 cellules observées.

Summary of publications investigating the chromium effect on translocation rate. Rate given for 1000 observed cells.

\begin{tabular}{lcccccc}
\hline $\begin{array}{c}\text { Référence de la } \\
\text { publication }\end{array}$ & $\begin{array}{c}\text { Taux de translocations } \\
\text { de la population } \\
\text { contrôle } \\
\text { Fréquence [IC 95 \%] }\end{array}$ & $\begin{array}{c}\text { Taux de translocations } \\
\text { de la population } \\
\text { exposée } \\
\text { Fréquence [IC 95 \%] }\end{array}$ & $\begin{array}{c}\text { Facteur } \\
\text { d'augmentation } \\
\text { (Significativité) }\end{array}$ & $\begin{array}{c}\text { Groupes } \\
\text { analysés }\end{array}$ \\
\hline $\begin{array}{l}\text { Maeng } \text { et al., 2004 } \\
\text { Non fumeurs a* }\end{array}$ & $4,58[1,84-9,44]$ & $19,62[11,99-30,30]$ & 4,3 & $(\mathrm{NS}) *$ & $\begin{array}{l}\text { Exposés : } \\
\text { Contrôles : }\end{array}$ & 9 \\
\hline $\begin{array}{l}\text { Maeng } \text { et al., } 2004 \\
\text { Fumeurs a* }\end{array}$ & $5,38[2,69-9,63]$ & $37,52[32,22-43,44]$ & 6,9 & $(p<0,05) *$ & $\begin{array}{l}\text { Exposés : } \\
\text { Contrôles : }\end{array}$ & 42 \\
\hline $\begin{array}{l}\text { Doherty } \text { et al., } \\
\text { 2001 b }\end{array}$ & $7,2[5,5-9,5]$ & $25,9[21,8-30,8]$ & 3,6 & $(p<0,0005)$ & $\begin{array}{l}\text { Exposés : } \\
\text { Contrôles : }\end{array}$ & 30 \\
\hline
\end{tabular}

NS : non significatif, a Les taux sont ramenés à l'ensemble du génome par la formule de Lucas. L'intervalle de confiance à $95 \%$ ([ ]) est calculé à partir du nombre de translocations observées et selon la table de la loi de Poisson. ${ }^{b}$ Valeurs et IC à $95 \%$ donnés pour 100 cellules observées au sein de la publication. * Test U-MannWhitney.

dehors de l'industrie, chez des personnes porteuses de prothèse orthopédique. L'étude du taux de translocations chez des personnes portant une prothèse depuis quelques années a été comparée à celui observé chez des personnes portant une prothèse implantée récemment (Doherty et al., 2001). En effet, au cours du temps, des débris de prothèse s'accumulent dans les tissus, se disséminent dans le corps et diffusent des métaux lourds. Une augmentation largement significative du taux de translocations est ainsi observée chez les personnes portant une prothèse ancienne par rapport au taux observé chez les personnes équipée d'une prothèse orthopédique plus récente (Doherty et al., 2001).

\section{Discussion}

La première partie a montré que les translocations pouvaient être générées par des facteurs biologiques ou des styles de vie. Cette deuxième partie de revue bibliographique s'est intéressée aux variations des translocations après exposition professionnelle à des facteurs génotoxiques. Dans cet objectif, ce papier fait la synthèse de publications étudiant l'impact de certains agents chimiques et certains métaux lourds sur la production des translocations. Il est important de soulever le fait que le nombre de publications qui étudie l'impact d'un facteur environnemental donné est assez faible, le nombre d'études n'excède pas 3 pour chaque facteur.

Les résultats de cette analyse bibliographique montrent que l'augmentation des translocations par un facteur toxique lié aux expositions professionnelles est plus marquée que par les agents dus au style de vie. Concernant 2 types de pesticides 
analysés dans ce papier, le nombre d'études est très faible. Nous obtenons une étude avec une augmentation significative des translocations pour les fongicides à EBDC et l'autre étude uniquement sur la phosphine ne montre pas d'effet. Le cas du benzène est particulier puisque les analyses n'ont tenu compte que des chromosomes impliqués dans des pathologies spécifiques de l'exposition. En revanche, quand les études s'intéressent aux translocations impliquant tous les chromosomes, aucune augmentation significative n'a été prouvée. Par conséquent les chromosomes étudiés sont importants dans ce type d'exposition. Le nombre de translocations est augmenté significativement souvent dans le cas de mélanges d'agents génotoxiques. Cela est le cas du mélange de composés HAP et de la synergie tabac et chrome. Par contre la sensibilité des translocations grâce à la technique FISH permet de mettre en évidence des différences d'exposition ou bien de protection vis-à-vis d'un agent toxique professionnel. En effet, la sensibilité de la technique FISH a souligné la nécessité d'équipements de protection adapté et ergonomique afin de réduire significativement le taux de translocations des individus exposés par rapport aux individus exposés sans protection. Cette technique peut donc être utile dans la surveillance des travailleurs exposés.

Cependant, le but de cette étude bibliographique est surtout la comparaison du taux de translocations engendré par divers agents par rapport à celui induit par l'irradiation. En effet nous cherchions à savoir si des agents autres que rayonnements ionisants étaient susceptibles de masquer les translocations dues à l'irradiation.

La figure 1 illustre cette comparaison, par l'étude de l'impact des facteurs étudiés sur le taux des translocations en fonction du type de facteur, de l'exposition et des études. Pour chaque étude le taux de translocations pour 1000 cellules de la population contrôle est indiqué en $\$$ et celui de la population exposée en 目. En outre, les taux de translocations obtenus au laboratoire de dosimétrie biologique de I'IRSN après irradiation in vitro d'échantillons de sang à des doses de 0,$2 ; 0,5$ et $0,7 \mathrm{~Gy}$, ont été aussi représentés sur cette figure, afin de pouvoir comparer les taux d'induction des translocations. En comparaison avec un groupe exposé aux rayonnements ionisants à une dose de $0,5 \mathrm{~Gy}$, le taux de translocations au sein des personnes exposées aux fongicides à EBDC est faible. On peut donc conclure que si un taux de translocation élevé est observé pour une personne exposée à la fois à cette catégorie de fongicides et aux rayonnements ionisants, il pourra n'être attribué qu'à l'irradiation. Cependant, lors d'une étude rétrospective de l'exposition aux rayonnements ionisants concernant des personnes fortement exposées au chrome ou aux hydrocarbures aromatiques, il sera difficile de différencier l'origine radiologique de l'origine toxicologique du taux élevé de translocations. Toutefois, les expositions aux hydrocarbures aromatiques ou au chrome ne sont pas courantes, et devraient pouvoir être identifiées par un 


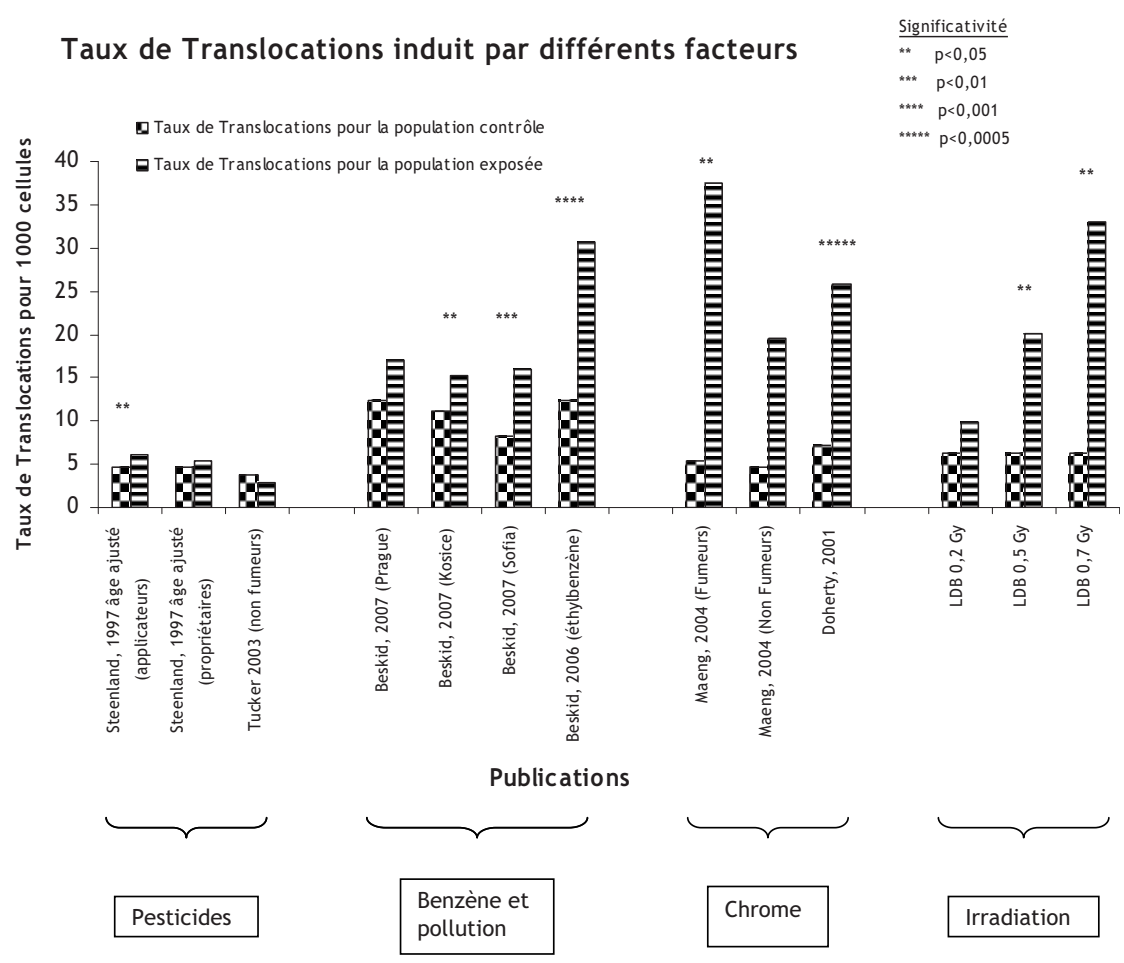

Les taux de translocations sont ceux obtenus dans les publications notées en abscisse $\mathrm{X}$. Les taux de translocations de l'irradiation sont ceux obtenus au laboratoire de Dosimétrie Biologique de L'IRSN pour des doses spécifiques.

Figure 1 - Comparaison entre les taux de translocations générés par différents facteurs et le taux de translocations générés par une irradiation in vitro.

Comparison between the translocation rate generated by different factors and that generated by irradiation in vitro.

questionnaire adapté décrivant entre autres les activités professionnelles actuelles et antérieures. Dans ce cas particulier, aucune conclusion sur une exposition radiologique éventuelle ne pourra être émise. Effectivement, établir la différence entre l'induction des translocations par les rayonnements ionisants et les autres expositions génotoxiques restera toujours difficile. Cependant un taux maximum de translocations pourrait être défini pour ce type d'exposition afin de pouvoir estimer le taux des translocations radio-induit.

Remerciements. Nous remercions vivement Murielle Gautier pour son aide précieuse sans laquelle la recherche bibliographique aurait été laborieuse. Nous remercions également Mr Jean-Marc Bertho pour ses conseils avisés. 


\section{RÉFÉRENCES}

Beskid O., Binkova B., Dusek Z., Rossner P., Solansky I., Kalina I., Zidzik J., Popov T.A., Farmer P.B., Sram R.J. (2007) Chromosomal aberrations by fluorescence in situ hybridization (FISH)Biomarker of exposure to carcinogenic PAHs, Mutat. Res. 620, 62-70.

Beskid O., Dusek Z., Solansky I., Sram R.J. (2006) The effects of exposure to different clastogens on the pattern of chromosomal aberrations detected by FISH whole chromosome painting in occupationally exposed individuals, Mutat. Res. 594, 20-29.

Bolognesi C. (2003) Genotoxicity of pesticides: a review of human biomonitoring studies, Mutat. Res. 543, 251-272.

Doherty A.T., Howell R.T., Ellis L.A., Bisbinas I., Learmonth I.D., Newson R., Case C.P. (2001) Increased chromosome translocations and aneuploidy in peripheral blood lymphocytes of patients having revision arthroplasty of the hip, J. Bone Joint Surg. 83, 1075-1081.

Forni A. (1996) Benzene-induced chromosome aberrations: a follow-up study, Environm. Health Perspect. 104 (Suppl 6), 1309-1312.

Garry V.F., Danzl T.J., Tarone R., Griffith J., Cervenka J., Krueger L., Whorton E.B. Jr., Nelson R.L. (1992) Chromosome rearrangements in fumigant appliers: possible relationship to nonHodgkin's lymphoma risk, Cancer Epidemiol. Biomarkers Prev. 1, 287-291.

Kim S.Y., Choi J.K., Cho Y.H., Chung E.J., Paek D., Chung H.W. (2004a) Chromosomal aberrations in workers exposed to low levels of benzene: association with genetic polymorphisms, Pharmacogenetics 14, 453-463.

Kim Y.K., Choi J.K., Cho Y.C., Eun J.H., Chung E.J., Paek D., Chung H.W. (2004b) Chromosomal aberrations in workers exposed to low levels of benzene: Association with genetic polymorphisms, Pharmacogenetics 14, 453-463.

Maeng S.H., Chung H.W., Kim K.J., Lee B.M., Shin Y.C., Kim S.J., Yu I.J. (2004) Chromosome aberration and lipid peroxidation in chromium-exposed workers, Biomarkers 9, 418-434.

Smith M.T., Zhang L., Wang Y., Hayes R.B., Li G., Wiemels J., Dosemeci M., Titenko-Holland N., Xi L., Kolachana P., Yin S., Rothman N. (1998) Increased translocations and aneusomy in chromosomes 8 and 21 among workers exposed to benzene, Cancer Res. 58, 2176-2181.

Sram R.J., Beskid O., Binkova B., Rossner P., Smerhovsky Z. (2004) Cytogenetic analysis using fluorescence in situ hybridization (FISH) to evaluate occupational exposure to carcinogens, Toxicol. Lett. 149, 335-344.

Sram R.J., Beskid O., Rössnerova A., Rössner P., Lnenickova Z., Milcova A., Solansky I., Binkova B. (2007) Environmental exposure to carcinogenic polycyclic aromatic hydrocarbons-The interpretation of cytogenetic analysis by FISH, Toxicol. Lett. 172, 12-20.

Steenland K., Cedillo L., Tucker J., Hines C., Sorensen K., Deddens J., Cruz V. (1997) Thyroid hormones and cytogenetic outcomes in backpack sprayers using ethylenebis(dithiocarbamate) (EBDC) fungicides in Mexico, Environ. Health Perspect. 105, 1126-1130.

Tucker J.D., Moore D.H. 2nd, Ramsey M.J., Kato P., Langlois R.G., Burroughs B., Long L., Garry V.F. (2003) Multi-endpoint biological monitoring of phosphine workers, Mutat. Res. 536, 7-14.

Zhang L., Rothman N., Li G., Guo W., Yang W., Hubbard A.E., Hayes R.B., Yin S., Lu W., Smith M.T. (2007) Aberrations in chromosomes associated with lymphoma and therapy-related leukemia in benzene-exposed workers, Environ. Mol. Mutagen. 48, 467-474. 